\title{
THE AVERAGE TEMPORAL AND SPECTRAL EVOLUTION OF GAMMA-RAY BURSTS
}

\author{
E. E. Fenimore \\ D436 Los Alamos National Laboratory, Los Alamos, NM 87545, USA \\ email: efenimore@lanl.gov \\ Received.........; accepted...........
}

\begin{abstract}
We have averaged bright BATSE bursts to uncover the average overall temporal and spectral evolution of gamma-ray bursts (GRBs). We align the temporal structure of each burst by setting its duration to a standard dura-

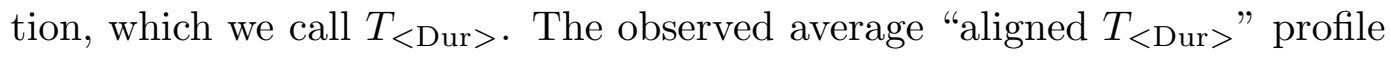
for 32 bright bursts with intermediate durations (16 to $40 \mathrm{~s}$ ) has a sharp

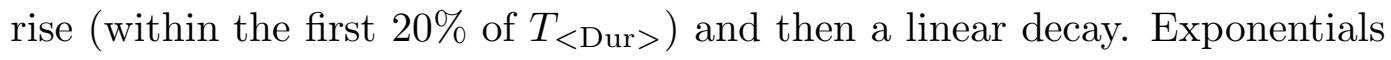
and power laws do not fit this decay. In particular, the power law seen in the x-ray afterglow $\left(\propto T^{-1.4}\right)$ is not observed during the bursts, implying that the x-ray afterglow is not just an extension of the x-ray evolution seen during the gamma-ray phase. The average burst spectrum has a low energy slope of -1.03, a high energy slope of -3.31 , and a peak in the $\nu F_{\nu}$ distribution at $390 \mathrm{keV}$. We determine the average spectral evolution. Remarkably, it is also a linear function, with the peak of the $\nu F_{\nu}$ distribution given by $\sim 680-600\left(T / T_{<\text {Dur }>}\right) \mathrm{keV}$. Since both the temporal profile and the peak energy are linear functions, on average, the peak energy is linearly proportional to the intensity. This behavior is inconsistent with the external shock model. Previously, trends have been reported for GRB evolution, but our results are quantitative relationships that models should attempt to explain.
\end{abstract} Subject Heading: Gamma-Ray, Bursts 


\section{INTRODUCTION}

Gamma-ray bursts (GRBs) are isotropically distributed yet are inhomogeneous implying that they are much farther away than typical galactic distance scales. One explanation is that they are at cosmological distances $(z \sim 1)$. At such distances, they require $\sim 10^{51}(\Omega / 4 \pi) \mathrm{erg} \mathrm{s}^{-1}$, where $\Omega / 4 \pi$ is the fraction of the sky radiated into by the bursts. There must exist a substantial energy reservoir to power GRBs, and it is often suggested that merging massive objects are the ultimate source of energy (Mészáros \& Rees 1993; Piran, Shemi, \& Narayan 1993; Rees \& Mészáros 1994).

GRBs are very diverse, with time histories ranging from as short as 50 $\mathrm{ms}$ to longer than $10^{3} \mathrm{~s}$. The long bursts often have very complex temporal structure with many subpeaks. Two classes of theories have arisen to explain how merging objects might produce the chaotic time history. In one theory (Mészáros \& Rees, 1993), there is a single release of energy ( $\sim 10^{53} \mathrm{erg}$ ) when two objects merge, which produces a relativistic shell. The observation of very high energy photons, coupled with the short time scales, indicates that the relativistic shell has a bulk Lorentz factor $(\Gamma)$ of $10^{2}$ to $10^{3}$. The shell expands for a rather long time (perhaps as long as $10^{7} \mathrm{~s}$ ). Eventually, the shell converts its bulk motion into gamma rays, perhaps due to relativistic shocks caused by sweeping up the interstellar medium (ISM). As the shell emits, the shell keeps up with the emitted photons, so they all arrive at a detector over a short period of time. The duration of the event is set by the expansion of the shell. If $t_{\mathrm{dur}}$ is the length of time that the shells emits, then the duration seen at a detector is $T_{\text {dur }}=t_{\text {dur }} /\left(2 \Gamma^{2}\right)$. In contrast, the complex temporal structure is due to inhomogeneities in the shell.

The alternative theory (Rees \& Mészáros 1994) is that the merger takes place over a period of time comparable to the observed duration of the burst. Perhaps the massive object is tidally disrupted, and the duration is set by the time it takes for most pieces to merge. Each subpeak in the GRB is the result of a separate explosive event at the central site. We refer to this as a "central engine." A large Lorentz factor is still required, thus each subpeak might produce a relativistic shell.

In both theories, the chaotic time history involves some randomness in the process. Neither involves a smooth process that produces a smooth 
envelope of emission. In this paper, we propose combining the time histories of many GRBs to average out the randomness and uncover the mean GRB envelope of emission.

\section{THE AVERAGE GRB ENVELOPE}

In the single relativistic shell scenario, the energy release is on a short time scale. If the shell expands for a period of time before it converts its energy to gamma rays, the curvature of the shell imposes a "FRED"-like shape to the time history. (Here, "FRED" is a fast rise, exponential decay, although the decay might not actually be exponential, it just falls faster than it rises.) The duration of the FRED is directly related to the radius of the shell at the time that it converts its energy (i.e., $2 \Gamma^{2} c T_{\text {dur }}$ ). This radius also could depend on the density of the ISM ( $\left.\rho_{\text {ISM }}\right)$ around the bursts. If all other things are equal, the observed burst duration from a single relativistic shell varies from burst to burst by some constant related to $\left(2 \Gamma^{2}\right)^{-1}$ and $\rho_{\text {ISM }}$. Thus, we scale the duration of each burst by a constant before averaging them. This can be viewed as an "aligned $T_{<\text {Dur }>}$ " average in contrast to the "aligned peak" averages, such as those used by Mitrofanov, et al. (1996). In the aligned peak average, each burst contributes to the average by aligning the largest peak. The time scale of the peak is conserved as it contributes to the average. In the aligned $T_{<\text {Dur }>}$ average, each burst contributes to the average by aligning the midpoint of the burst, and the time scale of the burst is adjusted to a standard duration which we call $T_{<\text {Dur }>\text {. }}$

The Burst and Transient Source Experiment (BATSE) catalog provides durations called $T_{90}$ and $T_{50}$ (Meegan et al. 1996). For example, $T_{90}$ is the duration which contains $90 \%$ of the counts. It is defined by finding the duration that excludes the first $5 \%$ and last $5 \%$ of the counts in the burst. There is a similar definition for $T_{50}$. Because of statistical variations in the background, it is impossible to determine exactly when the burst starts and stops. Thus, insisting on observing some small fraction (like 5\%) is more reliable than attempting to determine a duration which contains the whole event. We estimate an average duration, $T_{<\text {Dur }>}$, from $T_{90}$ and $T_{50}$. To first order, $T_{<\text {Dur }}$ is $T_{90} / 0.9$ or $T_{50} / 0.5$. By definition, the beginning point for $T_{90}$ or $T_{50}$ must be at a point at which the count rate is increasing. Thus, if we stretched each burst to a standard duration by scaling the time by some 
multiple of $T_{90}$, there would be a coherent peak at the first $5 \%$ point and at the last $5 \%$ point. Rather, we define $T_{<\text {Dur }}$ to be a combination of $T_{90}$ and $T_{50}$ to break up the coherency. Specifically, we define

$$
T_{<\text {Dur }>}=\frac{\left(T_{90}+T_{50}\right) / 2}{0.7}
$$

Although using the average tends to break up the coherency, some coherency will still remain and the average profile will tend to have a spike at the beginning. If GRBs had box-car like time profiles, then $T_{<\text {Dur }}>$ would be equal to $T_{90} / 0.9=T_{50} / 0.5$. In fact, GRB profiles usually decay after a fast rise such that $T_{90} / 0.9>T_{50} / 0.5$. As a result, $T_{<\text {Dur }>}$ is between $T_{90} / 0.9$ and $T_{50} / 0.5$.

We selected all 98 bursts from the BATSE 3B catalog that were longer than $1.5 \mathrm{~s}$ and brighter than 4 photons $\mathrm{s}^{-1} \mathrm{~cm}^{-2}$. To find the average profile of these events, we first defined the time at the middle of each event, $T_{\text {mid }}$, to be the average of the $T_{90}$ and $T_{50}$ midpoints. (The BATSE catalog provides these values, see Meegan et al. 1996.) We used the $0.064 \mathrm{~ms}$ BATSE data summed from 25 to $\sim 1000 \mathrm{keV}$ and rebinned each burst from $T_{\text {mid }}-T_{<\text {Dur }}$ to $T_{\text {mid }}+T_{<\text {Dur }>}$ into 200 time samples each $T_{<\text {Dur }>}^{-1}$ long. This produces a scaled time history from $T_{<\text {Dur }} / 2$ before the start of the $T_{<\text {Dur }}$ period to $T_{<\text {Dur }>} / 2$ after the end of the $T_{<\text {Dur }}$ period.

The bursts have peak fluxes that range up to $10^{2}$ photons $\mathrm{s}^{-1} \mathrm{~cm}^{-2}$. We normalize each burst so that they contribute to the average as if they were all at the same distance. This can be done in several ways. If the ultimate energy source is either neutron star-neutron star merger or neutron starblack hole merger, the total fluence is likely to be a standard candle. Figure 1a gives the average of the 98 bursts with the assumption that the fluence is a standard candle; that is, we normalized the total counts of a burst to unity before it contributed to the average. Alternatively, it is often assumed that the peak number flux is a standard candle. This is required when applied to studies involving the triggering because most detectors respond to peak counts. We think that it is an unlikely standard candle in scenarios involving merging objects. Nevertheless, for completeness, in Figure 1b we normalized the peak number flux of a burst to unity before it contributed to the average. Although our intent was to normalize in order to scale all bursts to the same 
distance, we do not know if GRBs have standard candle fluence, standard candle peak intensity, or if any characteristic is a standard candle. However, if no characteristic is a standard candle, we would still normalize the bursts such that no one burst would be allowed to dominate the average. The most likely way to avoid a single burst dominating would be to normalize by the total net counts. Thus, in the absence of a known standard candle, we would normalize as if the fluence was a standard candle.

Figure 1 shows bursts with $T_{90}$ durations from $1.5 \mathrm{~s}$ to $320 \mathrm{~s}$. Figure 2 gives the aligned $T_{<\text {Dur }}$ average time history using total fluence normalization for three ranges of durations: 1.5 to 16, 16 to 40, and 40 to $320 \mathrm{~s}$. Each group has about 33 bursts. If one averaged Figures $2 \mathrm{a}, \mathrm{b}$, and c, the result would be Figure 1a. For longer bursts, the trend is to have a larger initial emission relative to the rest of the burst. The most reliable average is probably the one that utilizes bursts with $T_{90}$ 's between 16 and $40 \mathrm{~s}$. Bursts with shorter durations often have only a few peaks, so the average profile can contain large variations. Also, short bursts do not have enough energyresolved samples (i.e., MER data) to study the evolution of the spectra over the $T_{<\text {Dur }}$ range (see Fig. $3 \mathrm{~b}$ below). Bursts with longer durations tend to be complex, making it difficult to recognize the beginning and end of the bursts. An additional reason to emphasize the 16 to $40 \mathrm{~s}$ range is that it covers the smallest dynamic range of durations (2.5 vs. 6.4 and 8.0 for the 1.5 to 16 and 40 to $320 \mathrm{~s}$ ranges, respectively). This is a reflection of the fact that the burst duration distribution peaks in the 16 to $40 \mathrm{~s}$ range so there are enough bursts to form an average even from a small range of durations. A small dynamic range means that the difference in stretching from one burst to another is small and, thus, the average should be more reliable.

For the reliable case of bursts with $T_{90}$ between 16 and $40 \mathrm{~s}$, the average profile appears to peak about $20 \%$ after the beginning of the $T_{<\text {Dur }}$ period and then decay linearly to the end of the $T_{<\text {Dur }}>$ period.

\section{AVERAGE TEMPORAL AND SPECTRAL EVOLUTION}

In Figure 3 we used the BATSE MER data to investigate the average spectral and temporal evolution. The BATSE MER data has 16 energy channels and $2.048 \mathrm{~s}$ time resolution. We used the 32 events with $T_{90}$ between 16 and $40 \mathrm{~s}$ because we consider that average time history to be the most 
reliable (see above). Figure $3 \mathrm{a}$ uses the same events as Figure 2b. In Figure 3a, the poorer time resolution of the MER data tends to wash out the large peak near $T_{<\text {Dur }}=0.2$. That peak is associated with the coherent increase that occurs where the average boundary of $T_{90}$ and $T_{50}$ occurs. The resulting average time history appears to rise to a peak and then fall linearly. We have fit a variety of temporal shapes to the decay portion between $20 \%$ after the beginning of the $T_{<\text {Dur }}>$ period to the end of the $T_{<\text {Dur }}>$ period. One cannot determine a strict goodness of fit (e.g., $\chi^{2}$ ) because the uncertainties are not the counting statistics (which, after all, are very good since we are adding together 32 bright bursts). Rather, the fluctuations are due to how the various peaks of 32 bursts add together. Presumably, if we had hundreds of bursts, the curve would be very smooth. However, we can use relative $\chi^{2}$ values to judge relative goodness-of-fit. We fit a linear function, an exponential function, and power law decays. We particularly checked if a $T^{-1.4}$ power law would fit because that type of decay is seen later during the x-ray afterglows (Piro et al. 1997). The linear fit was the best fit. The power law and exponential fit had $\chi^{2}$ values that were 2.2 and 3,8 , respectively, times larger and they disagreed with the observations in a systematic way, failing to agree with the observations at the ends of the time range. A power law with an index of -1.4 had a $\chi^{2}$ that was 6.4 times larger than the linear fit. The best linear function is:

$$
\begin{array}{rlrl}
I & =5.56 \frac{T}{T_{<\text {Dur }}} & \text { if } T<0.18 T_{<\text {Dur }} \\
& =1.19-1.06 \frac{T}{T_{<\text {Dur }>}} \quad \text { if } T>0.18 T_{<\text {Dur }>} .
\end{array}
$$

We calculated aligned $T_{<\text {Dur }}>$ averages for each of the 16 MER energy channels. From these, six spectra were formed, each covering $15 \%$ of the $T_{<\text {Dur }}$ range. The first one started $5 \%$ after the beginning of the $T_{<\text {Dur }}$ range, and the last one ended at $5 \%$ before the end of the $T_{<\text {Dur }>}$ range. For each of these spectra, we fit the "Band" spectral shape (Band et al., 1993). This shape consists of a low energy slope $(\alpha)$, the peak of the $\nu F_{\nu}$ distribution $\left(E_{p}\right)$, and a high energy slope $(\beta)$. We first fit the Band shape to the sum of all six spectra. This yielded an overall average GRB spectra. The parameters are $\alpha=-1.03, E_{p}=390 \mathrm{keV}$, and $\beta=-3.13$. Since this is 
an average of 32 bright GRBs, it ought to provide a fairly good average GRB spectra. To investigate the average spectral evolution, we analyzed each of the six spectra separately, fixing $\alpha$ to -1.03 and $\beta$ to -3.13 . Thus, the only free parameter is $E_{p}$. Figure $3 \mathrm{~b}$ shows the resulting spectral evolution. It is a remarkably straight line:

$$
E_{p}=680-600 \frac{T}{T_{<\text {Dur }>}} \mathrm{keV}
$$

\section{DISCUSSION}

We have averaged bright BATSE bursts to uncover the average overall temporal and spectral evolution of GRBs. The expected profile from a single relativistic shell that expands in a photon quiet phase and then becomes gamma-ray active (i.e., the external shock model) should have an envelope with a fast rise and and a power law decay phase (i.e., $T^{-\alpha-1}$, where $\alpha$ is a typical number spectral index for GRBs, say $\sim 1.5$ ). The peak of $\nu F_{\nu}$ should evolve as $T^{-1}$ (Fenimore, Madras, \& Nayakshin 1996; Fenimore \& Sumner 1997). Figure 3a gives the observed average temporal profile for 32 bright bursts and a power law decay is inconsistent with it. Figure $3 \mathrm{~b}$ gives the average spectral evolution, and it is inconsistent with a $T^{-1}$ decay. We conclude that the average burst envelope does not support the external shock model. This disagreement adds to the arguments that the external shock model cannot explain the observed GRB time histories unless the shell has structure with angular scales much smaller than $\Gamma^{-1}$ (see Fenimore, Madras, \& Nayakshin 1996; Fenimore, Ramirez, \& Sumner 1998).

The fact that the decay during the gamma-ray phase is inconsistent with the decay during the x-ray afterglow (i.e., $T^{-1.4}$, see Costa et al. 1997) argues that the x-ray afterglow is not just a continuation of the evolution of the x-rays seen during the GRBs.

The observed decay is quite interesting. Both the average temporal evolution and the average spectral evolution are linear functions. Thus, in the decay phase, the intensity is linearly proportional to the peak of the $\nu F_{\nu}$ distributions. The $\nu F_{\nu}$ evolution is effectively linear over the full duration of the event. In particular, the first point in Figure $3 \mathrm{~b}$ occurs during the rise in the time history. This is consistent with Norris et al. (1996) which found 
that the the hardness often peaks before the peak in the time history. Here, rather than just reporting a trend, we establish a quantitative relationship between the time history and spectral evolution.

Something in the physics determines the duration of events. In the context of the external shock models, variations in $\Gamma$ from burst to burst can easily cause the bursts observed in arrival time to have different durations. We have argued that the GRB phase is not a single relativistic shell, as in the external shock model (Fenimore, Madras, \& Nayakshin 1996, Fenimore, Ramirez, \& Sumner 1998, and the results of this paper). Rather, the GRB phase requires a central engine with energy releases lasting up to $>100 \mathrm{~s}$ or the single relativistic shell must have structure much smaller than $\Gamma^{-1}$. For example, this "central engine" might be caused by internal variations of $\Gamma$ in a relativistic wind. It is not clear why these variations should release energy such that, on average, the observed counts decreases linearly and the peak of the energy spectrum also varies linearly. The processes are random, and the peaks that are produced vary substantially in intensity. The result is that no one burst shows the underlying linear relationships. When many bursts are added together, the hidden pattern is revealed.

Although we have only shown the linear relationships in the context of an average of many bursts, it seems reasonable that this pattern exist in most of the bursts but is hidden by the chaotic peak structure. However, some bursts seem to show a different pattern. In an earlier report on this topic (Fenimore \& Sumner 1997), we presented results like these shown in Figure 3 for fewer bursts, where the linear trends were not as clear. We also did an aligned $T_{<\text {Dur }>}$ average for six FRED-like bursts (BATSE numbers 467, 543, $678,2431,2736$, and 2994). Only one of these bursts were included in Figure 3 , the rest were too weak to meet our criterion. In the case of the FRED-like bursts, a power law decay similar to that expected from an external shock model was observed for the time history but not for the spectral evolution (Fenimore \& Sumner 1997).

Two other quantitative relationships have been claimed for GRB evolution. Liang \& Kargatis (1996) suggested that $E_{p}$ varies as $\exp ^{-\Phi(T) / \Phi_{0}}$, where $\Phi(T)$ is the photon fluence up to time $T$. Given our equation (2), $\Phi(T)$ would scale as $1.19 T-0.53 T^{2}$. Thus, we do not see the Liang \& Kar- 
gatis pattern in the aligned $T_{<\text {Dur }}$ average profiles. The other quantitative pattern concerns how the duration of peaks varies with energy. Peaks are broader at lower energy, and Fenimore et al. (1995) found that the GRB time structure scales as $E^{-0.45}$. We have combined equations (2) and (3) to characterize the width of the time structure as a function of energy. No pattern was evident; the aligned $T_{<\text {Dur }}$ linear relationship is not the same as the aligned peak relationship. Thus, if the physics is such that the aligned peak average is relevant, then models must explain why the time structure scales as $E^{-0.45}$. If the physics is such that the aligned $T_{<\text {Dur }>}$ average is relevant, then models must explain why the time histories and spectral evolution vary linearly with the duration of the events. Both relationships should be consistent with the models since the aligned peaks tend to be a feature of the largest peak, while the aligned $T_{<\text {Dur }}$ is a feature of the whole burst.

We thank Jay Norris for providing the MER data.

\section{REFERENCES}

Band, D. L., et al. 1993, ApJ, 413, 281

Costa, et al., 1997, Nature, 387, 783

Fenimore, E. E., Madras, C. D., \& Nayakshin, S., 1996, ApJ 473, 998, astro-ph/9607163

Fenimore, E. E., \& Sumner, M. C., 1997, All-Sky X-Ray Observations in the Next Decade, eds. Matsuoka, M., \& Kawai, N., in press, astroph/9705052

Fenimore, E. E., in't Zand, J. J. M., Norris, J. P., Bonnell, J. T., \& Nemiroff, R. J., 1995, ApJ 448, L101, astro-ph/950407

Fenimore, E. E., Ramirez, E., \& Sumner, M. C., Gamma-Ray Bursts: 4th Huntsville Symposium, eds. Meegan, Preece, \& Koshut, in press, astroph/9712303.

Liang, E. P., \& Kargatis, V. E., 1996, Nature, 381, 49

Meegan, C. A., et al. 1996, ApJS, 106, 65

Mészáros P., \& Rees, M. J., 1993, ApJ, 405, 278

Mitrofanov, I. G., et al., 1996, ApJ, 459, 570.

Norris, J. P., et al. 1996, ApJ, 459, 393.

Piran, T., Shemi, A., \& Narayan, R. 1993, MNRAS, 236, 861

Piro, L., et al. 1997, A\&A, in press 
Rees, M. J., \& Mészáros , P., 1994, ApJ, 430, L93

\section{Figure Captions}

Figure 1: Average envelope of gamma-ray emission from 96 bright BATSE bursts. GRB models involving a single relativistic shell release energy at the central site very quickly, and the duration of a GRB is set by the evolution of the relativistic shell. The emission from the shell appears contracted in time due to relativistic effects. To find the average time history envelope of emission in this case, we scale the observed duration of each GRB ("T $T_{<\text {Dur }}$ ") to a standard duration. This could account for different bulk Lorentz factors of the shells.

(a) Each burst was normalized to the same distance by assuming a standard candle total fluence.

(b) Each burst was normalized to the same distance by assuming a standard candle peak counts.

Figure 2: Average envelope of gamma-ray emission for three ranges of observed durations. Each burst was normalized to the same distance by assuming a standard candle total fluence, and each duration was scaled to a standard duration (" $T_{<\text {Dur }}$ "). The average profile for bursts with duration between 16 and $40 \mathrm{~s}$ (b) is probably the most reliable "aligned $T_{<\text {Dur }}$ " average profile.

Figure 3: The average temporal and spectral evolution of bright events with intermediate durations ( $T_{90}$ between 16 and $40 \mathrm{~s}$ ) based on the BATSE MER data.

(a) The average time history. The decay phase starting $20 \%$ after the beginning of the $T_{<\text {Dur }}$ period is inconsistent with exponential decays and power law decays (including the $T^{-1.4}$ decay seen in the x-ray afterglow phase). Instead, the decay is consistent with a linear slope.

(b) The average spectral evolution. The spectral evolution is found by fix-

ing the low energy and high energy slopes at the average for the bursts and allowing only the peak of the $\nu F_{\nu}$ to vary. The peak energy is also a linear function. Thus, on average, the intensity is a linear function of the peak of the $\nu F_{\nu}$ distribution. This pattern is inconsistent with that expected from the external shock model. 
Fig. 1

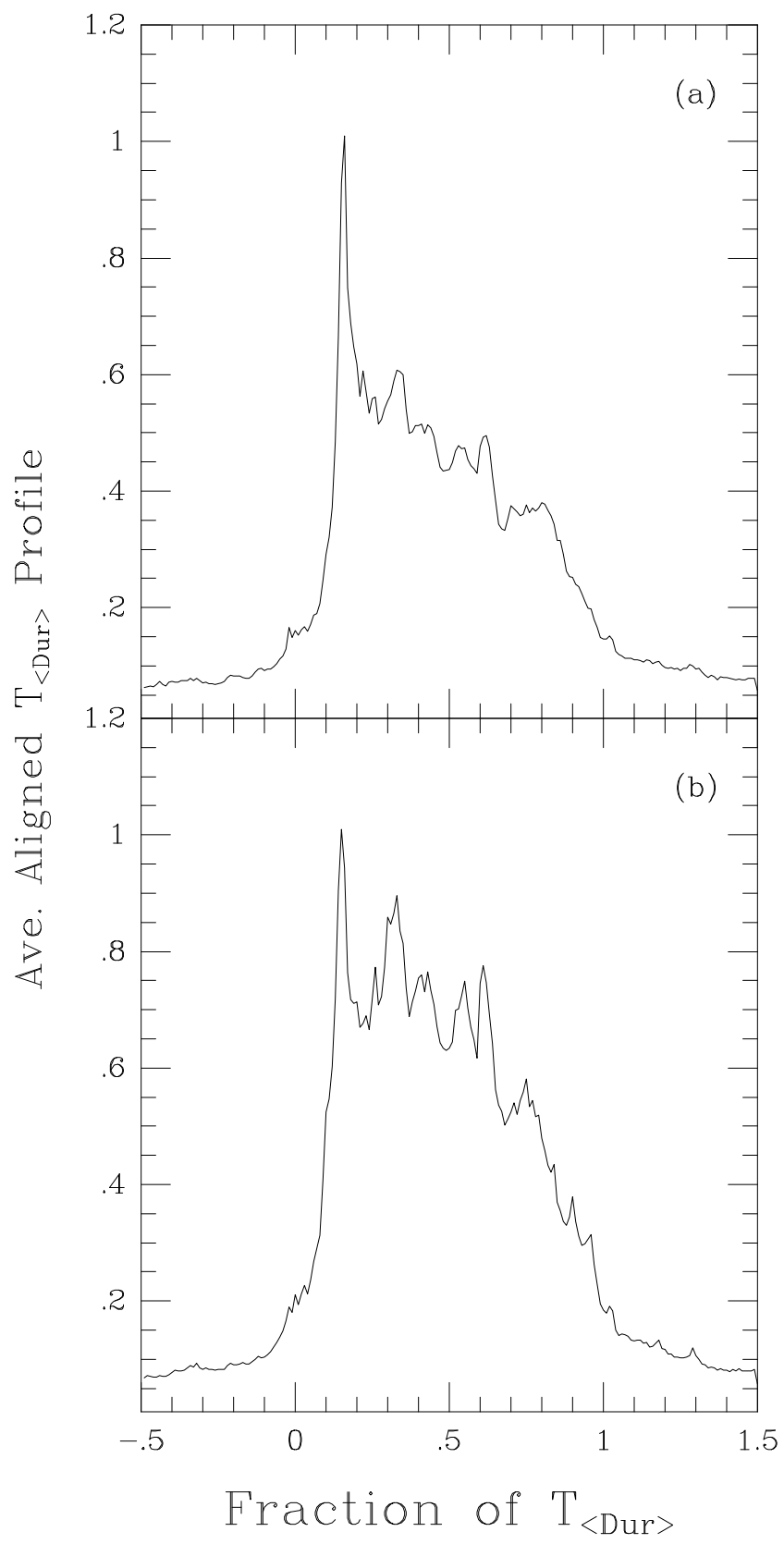


Fig. 2

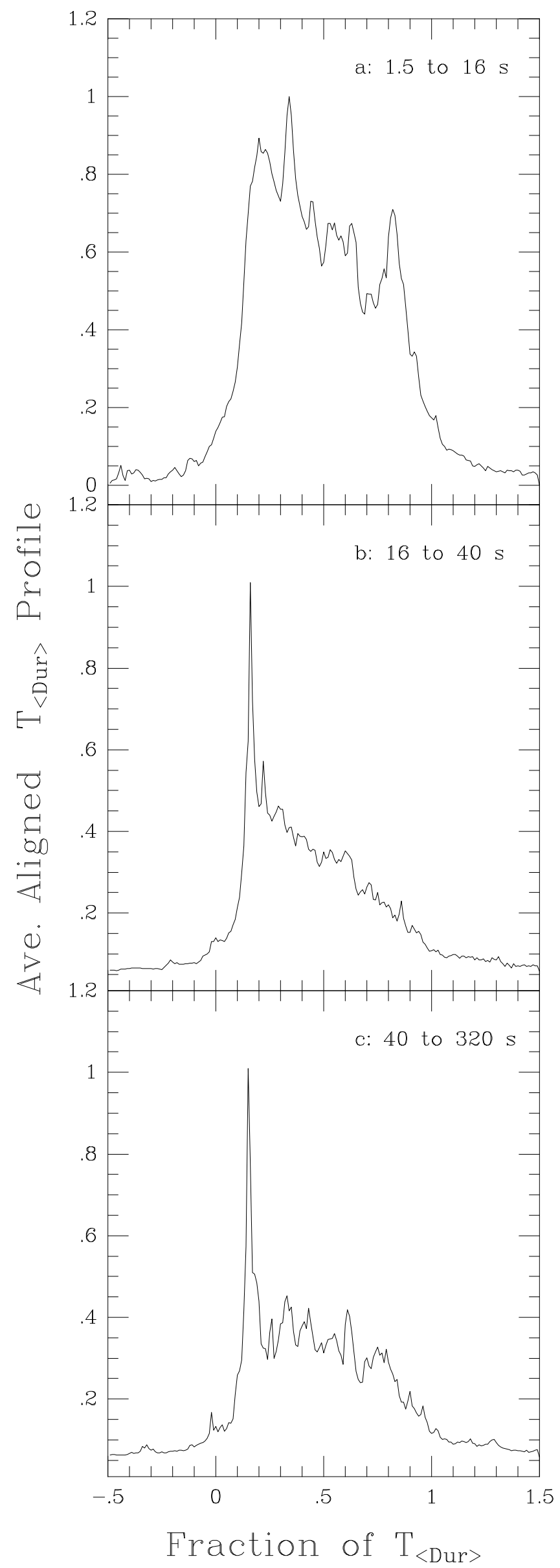




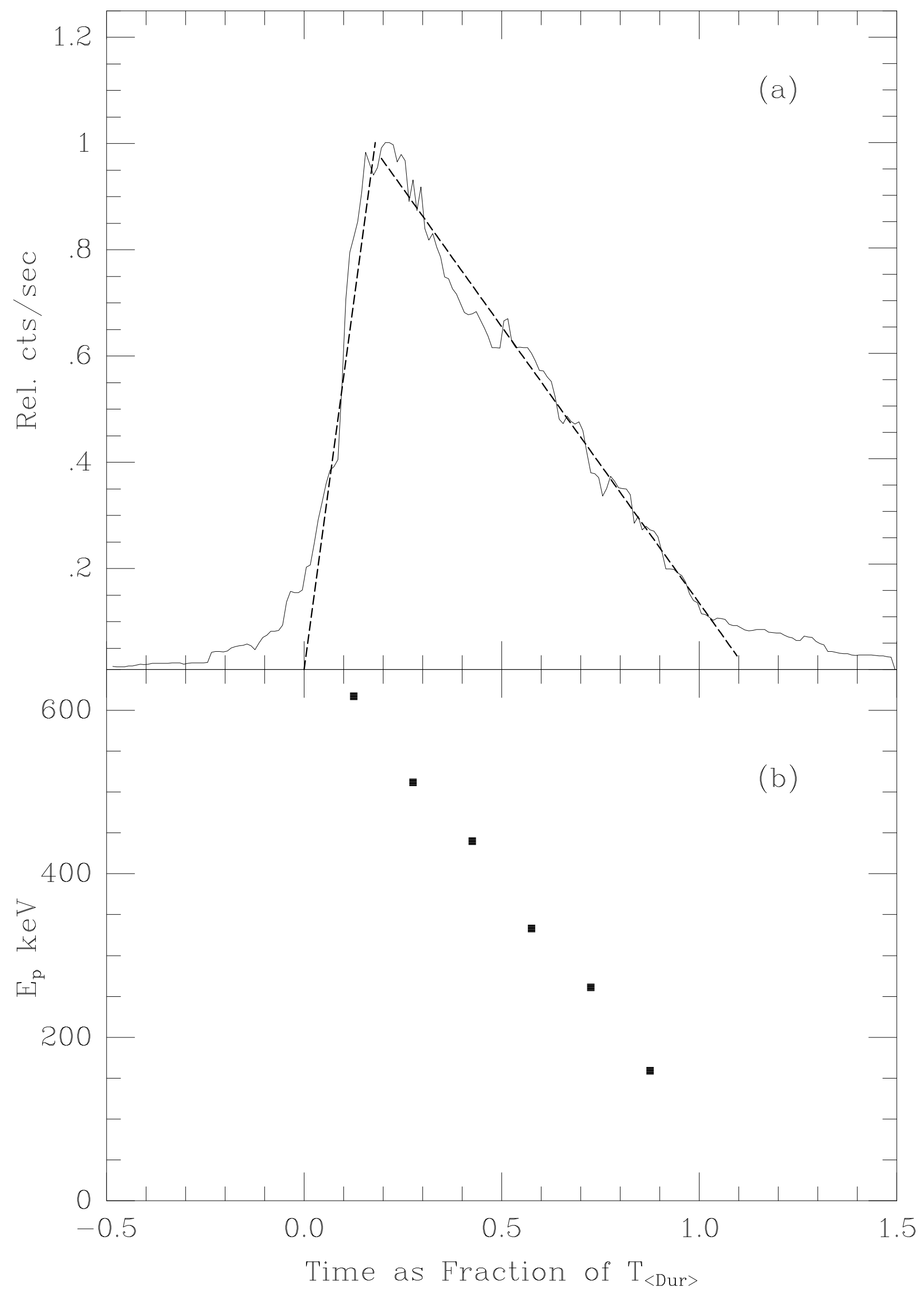

